\title{
Spacer Oligonucleotide Typing
}

National Cancer Institute

\section{Source}

National Cancer Institute. Spacer Oligonucleotide Typing. NCI Thesaurus. Code C85843.

A PCR-based method for differentiating M. tuberculosis strains based on amplification of the polymorphic direct repeat locus. 